\title{
RESEARCH HIGHLIGHT Towards precise, safe genome editing
}

\author{
Sebastian Memczak ${ }^{1,2}$, Yanjiao Shao ${ }^{1,3}$ and Juan Carlos Izpisua Belmonte (D) \\ Cell Research (2019) 29:687-689; https://doi.org/10.1038/s41422-019-0188-x
}

\begin{abstract}
Commonly used Cas9:gRNA programmed base-editor complexes introduce frequent, so far unrecognized RNA off-target edits. Novel, engineered enzymes may overcome this hurdle towards clinical applications of genome engineering.
\end{abstract}

CRISPR/Cas-mediated DNA base editing is a recently developed technology that holds the promise to be a future therapeutic for human genetic diseases. The approach is based on elegantly engineered nucleotide-modifying enzymes that, when fused to catalytically inactive or single strand cleaving Cas variants can be programmed by guide RNAs (gRNA) to target specific genomic loci and introduce or repair single-base mutations. ${ }^{1}$

Early CRISPR-Cas9 genome editing tools rely on the introduction of DNA double strand breaks (DSBs) by Cas9-mediated endonucleolytic cleavage. Subsequent cellular repair via non-homologous end joining results in insertion or deletion of one to a few bases at the targeted locus. Alternatively, the lesion can be patched by homology directed repair. Both pathways can be utilized for genome engineering including the introduction of exogenous DNA.

CRISPR-guided base editing, in contrast, converts DNA nucleotides without the introduction of DSBs and is thought to largely avoid associated cellular stress and error-prone repair (Fig. 1). At the same time it enables direct installation of transition mutations at targeted loci independent of exogenous DNA donor templates and therefore holds great promise for broad application in basic research and eventually clinics. In principle, base editing could be efficiently used to install hundreds of disease-correcting or disease-suppressing mutations in vitro and in vivo and seems to yield predictable outcomes. ${ }^{2}$ Therefore, CRISPR-guided base editing is particularly attractive.

A recent publication in Nature, however, uncovered that commonly used cytosine base editors (CBEs) as well as adenine base editors (ABEs) introduce a severe and so far unrecognized adverse effect: frequent, wide-ranging and gRNA-independent offtarget edits in RNA. ${ }^{3}$ Considering that originally a CBE was identified as a RNA-modifying enzyme, ${ }^{4}$ the authors decided to test whether its activity is retained in protein fusion complexes used in current genome editing experiments. They transfected BE-GFP constructs and subsequently sorted human cells with highest GFP expression and analyzed genome and possible transcriptome editing. By using multiple independent gRNAs, they observed not only desired DNA edits at the targeted loci as expected in the transfected HepG2 hepatocarcinoma and HEK293T cells, but also RNA edits in $~ 50 \%$ of the expressed genes, almost all of which were C-to-U edits and not due to changes in DNA and not observed in control cells. Further computational analysis showed that all regions of expressed RNAs including the protein coding sequence were affected, an observation that suggests widespread proteome changes if these RNAs are translated. Therefore, cellular behavior could be altered by base editing in unintended and unpredictable ways. Unlike previously described permanent and sparse DNA off-targets, however, these RNA edits are widespread, manifold and due to the short half-life of RNA, transient. In the current study the viability of cells subjected to base editing was found mildly altered, and any potential long-term effects of RNA off-targeting in vitro and in vivo remain to be investigated.

A more efficient alternative to $C B E s$ are adenine deaminating $A B E s^{5}$ that convert A-T to G-C base-pairs. This family of editors elicits dramatically less DNA off-targets than $\mathrm{CBEs}^{6,7}$ however, in transfected HEK293T cells, ABE also exhibited transcriptome-wide unspecific RNA editing well above the background level of endogenous adenosine deamination. ${ }^{3,8}$ Therefore, this phenomenon needs to be considered a general problem of current base editing approaches.

In most genome editing studies, inadvertent DNA alterations at non-targeted loci were probed using targeted or whole genome sequencing. In addition, large on-target rearrangements ${ }^{9}$ and general immunoreactions can also hamper DNA editing specificity and hinder its clinical application. ${ }^{10}$ Previously, off-target of BEs effects were described as: (1) unspecific gRNA-dependent or -independent editing on DNA; (2) multiple edits within the ontarget editing window. ${ }^{1,6,7}$ The off-target RNA editing discussed herein is an important extension to the previous findings. It is important to note that precise quantification of base edits is challenging and can vary substantially between experimental and analytical strategies and may depend largely on BE expression duration and level. Irrespective of these technical complications, the scale of the RNA edits observed here is extensive and makes these findings very noticeable to the field.

The results vividly illustrate the current limitations of the base editing approach, but further engineering improved enzymes and protocol optimization are promising to mitigate or overcome those shortcomings. The authors offer engineered CBEs-selective curbing of unwanted RNA editing (SECURE) as one potential solution: previously reported $\mathrm{CBE}$ variants which showed mitigated C-to-U editing on RNA while maintaining DNA editing activity. Recent structure-guided mutation of deaminase domains of $A B E s$ likewise yielded improved specificity of adenosine base editing. $^{8}$ Besides screening or evolving of fidelity-optimized enzymes, time- or spatially-constrained expression or activity of $\mathrm{BEs}$, sgRNA tuning ${ }^{11}$ the incorporation of split-enzyme designs ${ }^{12}$ or novel DNA-specific functional domains could be instrumental to raise both efficiency and specificity. Additionally, the efficient delivery of $\mathrm{BEs}$ in vivo by currently pursued transient overexpression, complexed protein introduction or viral transduction remains a technical challenge and will almost certainly produce

${ }^{1}$ Gene Expression Laboratory, Salk Institute for Biological Studies, 10010 North Torrey Pines Road, La Jolla, CA 92037, USA; ${ }^{2}$ Paul F. Glenn Center for Biology of Aging Research at

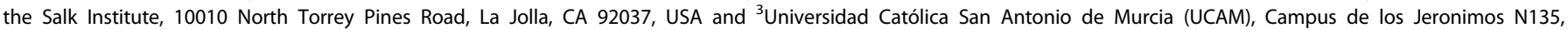
12 Guadalupe, CA 30107, Spain

Correspondence: Juan Carlos Izpisua Belmonte (belmonte@salk.edu)

Published online: 14 June 2019 

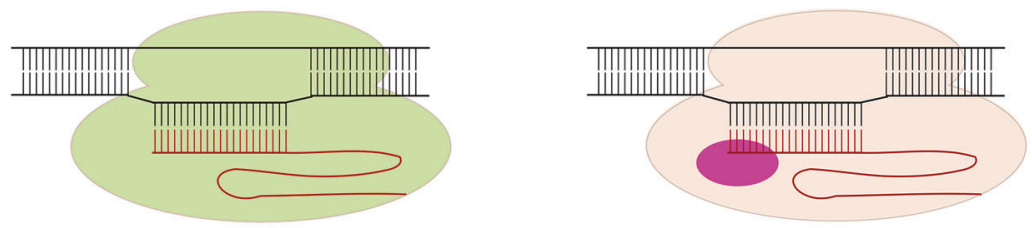

double strand break

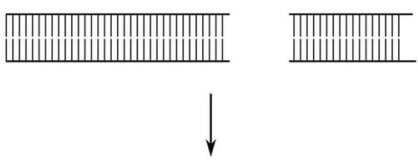

random indels

NHEJ

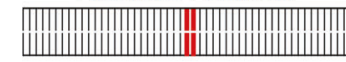

precise insertion

HDR

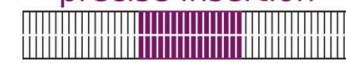

deamination
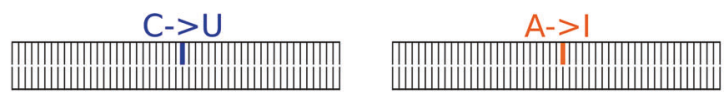

$\downarrow$

$\mathrm{T}$
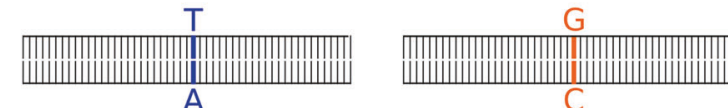

\section{b DSB off-targets}

1) unspecific DNA targets

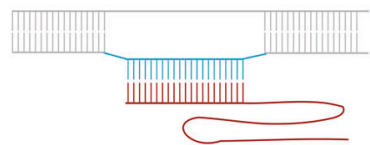

2)

p53 activation

\section{p53}

3)

cellular stress

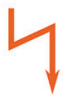

4)

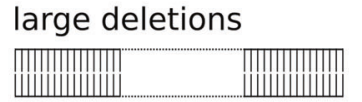

\section{c Base editing off-targets}

1)

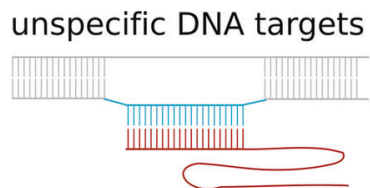

2) multiple edits
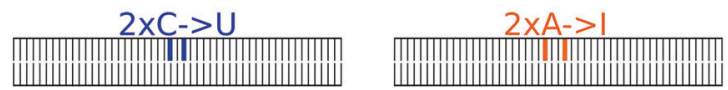

3)

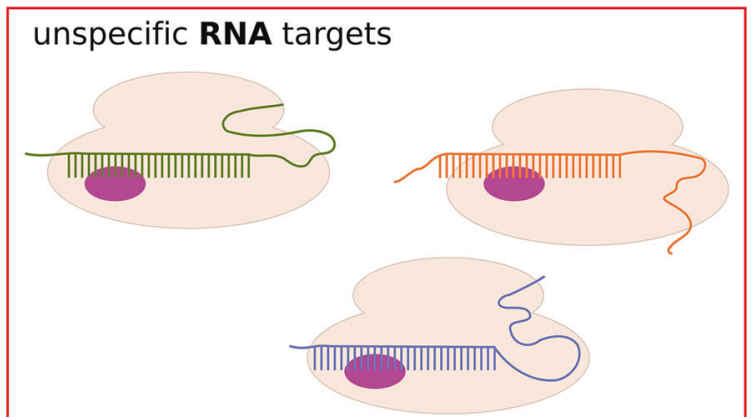

Fig. 1 On- and off-targets of CRISPR/Cas9-mediated genome editing. a CRISPR/Cas9 complexes are recruited to genomic DNA by gRNAs (red). Active Cas9 (green complex, left side) can induce double strand breaks which are repaired by cellular non-homologous end joining (NHEJ), creating indels or by a homology directed repair (HDR) mechanism. Using catalytically inactive or strand-nicking Cas9 (orange, right side) fused to base editors (purple complex subunit), cytosines and adenines can be edited by deamination to create or repair point mutations. b Known off-targets of fully active Cas9 include recruitment to genomic sites with only partial gRNA complementarity (bright blue), p53 pathway activation, induction of a genomic stress response and deletions or other large-scale re-arrangements at the on-target site. c Base editor complexes may be inadvertently directed to DNA off-target sites as well and can edit more than the intended bases in close proximity to the target. Importantly, frequent, wide-spread and gRNA-independent editing of RNA was recently described in cells treated with commonly used base editors. ${ }^{3}$ 
side-effects. We consider the fundamental problem of $\mathrm{BE}$ specificity, however, as currently most urgent. An interesting complication arises from the observed difference in off-target frequencies between in vitro and in vivo systems, and understanding the underlying molecular mechanisms may hold lessons for successful application of BEs in the future.

Given the current innovation rate of the field, one should be optimistic that the technology of DNA base editors will fulfill their promises when guided by incremental engineering and thorough evaluation as exemplified by the work discussed here.

\section{ACKNOWLEDGEMENTS}

Work in the laboratory of J.C.I.B. was supported by the Glenn Foundation and Universidad Católica San Antonio de Murcia (UCAM).

\section{REFERENCES}

1. Komor, A. C., Kim, Y. B., Packer, M. S., Zuris, J. A. \& Liu, D. R. Nature 533, 420-424 (2016).

2. Rees, H. A. \& Liu, D. R. Nat. Rev. Genet. 19, 770-788 (2018).

3. Grünewald, J. et al. Nature 569, 433-437 (2019).

4. Teng, B., Burant, C. F. \& Davidson, N. O. Science 260, 1816-1819 (1993).

5. Gaudelli, N. M. et al. Nature 551, 464-471 (2017).

6. Zuo, E. et al. Science 364, 289-292 (2019).

7. Jin, S. et al. Science 364, 292-295 (2019).

8. Rees, H. A., Wilson, C., Doman, J. L. \& Liu, D. R. Sci. Adv. 5, eaax5717 (2019).

9. Kosicki, M., Tomberg, K. \& Bradley, A. Nat. Biotechnol. 36, 765-771 (2018).

10. Charlesworth, C. T. et al. Nat. Med. 25, 249-254 (2019).

11. Kocak, D. D. et al. Nat. Biotechnol. 37, 657-666 (2019).

12. Zetsche, B., Volz, S. E. \& Zhang, F. Nat. Biotechnol. 33, 139-142 (2015). 